\title{
Aumento de matéria orgânica num latossolo bruno em plantio direto
}

\author{
No-tillage increases soil organic matter in a South Brazilian oxisol
}

\author{
Falberni de Souza Costa ${ }^{1}$ Cimélio Bayer ${ }^{2}$ Jackson Adriano Albuquerque $^{3}$ \\ Sandra MaraVieira Fontoura ${ }^{4}$
}

\begin{abstract}
O aumento do estoque de matéria orgânica do solo em sistemas conservacionistas de manejo é dependente do tipo de solo e das condições climáticas, e tem reflexos na qualidade física do solo. Neste estudo, avaliou-se um experimento de longa duração (21 anos) quanto ao efeito do sistema plantio direto (PD) sobre os estoques de carbono

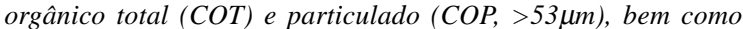
a sua relação com a estabilidade de agregados de um Latossolo Bruno, em Guarapuava, PR. O solo em PD apresentou taxa de incremento de $0,15 \mathrm{Mg} \mathrm{ha}^{-1}$ ano $^{-1}$ de COT e 0,06Mg ha $\mathrm{M}^{-1}$ ano $^{-1}$ de COP na camada de 0-20 cm, as quais foram calculadas em comparação aos estoques de carbono orgânico do solo em preparo convencional. As baixas taxas de incremento nos estoques de carbono orgânico possivelmente estejam relacionadas à alta estabilidade física da matéria orgânica neste solo argiloso e com mineralogia predominantemente gibsítica. $O$ diâmetro médio geométrico (DMG) dos agregados de solo variou de 1,6 a 3,7mm e foi positiva e linearmente relacionado com os teores de COT e COP, o que reforça a importância da matéria orgânica na qualidade física de Latossolos subtropicais.
\end{abstract}

Palavras-chave: manejo conservacionista, matéria orgânica particulada, agregação.

\section{ABSTRACT}

The increase of soil organic matter stocks under conservation management systems is dependent of soil type and regional climatic conditions, and has influence on the physical quality of soils. In this study the long-term (21 yr) effect of no-tillage on total soil organic carbon (TOC) and particulate organic carbon $(P O C,>53 \mu \mathrm{m})$, as well as its relationship with aggregate stability of a South Brazilian Oxisol (Haplohumox), was evaluated. In comparison with TOC stocks of conventionally-tilled soil, no-tillage soil had a net storage of $0.15 \mathrm{Mg} \mathrm{ha} a^{-1} y r^{-1}$ of TOC, and $0.06 \mathrm{Mg} \mathrm{ha}^{-1} \mathrm{yr}^{-1}$ of POC, in the $0-20 \mathrm{~cm}$ soil layer. These low accumulation rates of organic carbon probably were due to the high clay content and gibbsitic mineralogy of the soil, which determine a great physical stability of soil organic matter. The mean geometric diameter (MGD) of soil aggregates varied from 1.6 to $3.7 \mathrm{~mm}$, and had a positive relationship with TOC and POC, reinforcing the crucial role of organic matter on physical quality of subtropical oxisols.

Key words: conservation management systems, soil organic carbon, soil aggregation.

Sistemas conservacionistas de manejo apresentam um expressivo efeito na melhoria da qualidade de solos tropicais e subtropicais. O plantio direto aumenta os estoques de matéria orgânica e a estabilidade de agregados, sendo a magnitude deste efeito dependente do tipo de solo e condições climáticas (BALESDENT et al., 2000). Frações lábeis da matéria orgânica normalmente têm influência maior sobre a agregação do solo, devido tratar-se de uma fonte mais facilmente assimilável de $\mathrm{C}$ e de energia pelos microrganismos heterotróficos (CHAN, 1997), cujos compostos do metabolismo microbiano atuam na estabilização de macroagregados de solo. O objetivo deste estudo foi avaliar o efeito do sistema plantio direto sobre os estoques de carbono orgânico

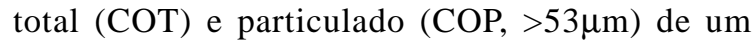
Latossolo Bruno alumínico câmbico, em comparação ao sistema de preparo convencional (PC). Avaliou-se

\footnotetext{
${ }^{1}$ Engenheiro Agronômo, MSc, doutorando no Programa de Pós-grduação em Ciência do Solo, Universidade Federal do Rio Grande do Sul (UFRGS), Porto Alegre, RS. E-mail: falberni.costa@ufrgs.br

${ }^{2}$ Engenheiro Agronômo, Doutor Professor, Depto de Solos, UFRGS, RS. E-mail: cimelio.bayer@ufrgs.br. Autor para correspondência. ${ }^{3}$ Engenheiro Agronômo, Doutor Professor do Departamento de Solos, Universidade do Estado de Santa Catarina, Lages, SC. Email: a2ja@cav.udesc.br

${ }^{4}$ Engenheiro Agronômo, MSc, Pesquisador da FAPA-Fundação Agrária de Pesquisa Agropecuária, Guarapuava, PR. E-mail: sandrav@agraria.com.br
} 
também o diâmetro médio geométrico (DMG) dos agregados, a fim de se verificar a relação da matéria orgânica com a estabilidade de agregados neste solo.

$\mathrm{O}$ estudo foi realizado em experimento de longa duração (21 anos), instalado em 1978, na área da Fundação Agrária de Pesquisa Agropecuária (FAPA), Guarapuava, PR. Em amostras de solo coletadas em 1999, nas profundidades de 0-5, 5-10 e 10-20cm, determinou-se o COT (EMBRAPA, 1997) e o DMG (KEMPER \& CHEPIL, 1965). O carbono orgânico particulado (COP) foi fracionado a partir da mistura de $20 \mathrm{~g}$ de solo $(2 \mathrm{~mm})$ e $60 \mathrm{ml}$ de hexametafosfato de sódio (5 $\left.\mathrm{g} \mathrm{L}^{-1}\right)$, agitação por 16 horas, e passagem da suspensão em peneira de $53 \mu \mathrm{m}$ (CAMBARDELLA \& ELLIOT, 1992). O material orgânico retido na peneira consiste no COP, o qual foi quantificado a partir da massa da fração seca em estufa a $60^{\circ} \mathrm{C}$, e análise do teor de carbono orgânico (EMBRAPA, 1997). As concentrações (massa/volume) e quantidades $\left(\mathrm{Mg} \mathrm{ha}^{-1}\right)$ de COT e COP foram calculadas levando-se em consideração os valores de densidade do solo a campo, determinadas nas respectivas profundidades do solo. Os resultados de COT e COP foram submetidos à análise de variância, sendo a diferença entre médias avaliada pelo teste de Tukey a 5\% de probabilidade de erro. A relação entre o DMG e o COT e COP foi avaliada pela significância dos coeficientes de determinação de regressões lineares.

A partir do rendimento acumulado das culturas $\left(104,1 \mathrm{t} \mathrm{ha}^{-1}\right.$ no PC e $123,6 \mathrm{t} \mathrm{ha}^{-1}$ no PD) nas 42 safras (inverno e verão), e considerando-se um índice de colheita aparente médio de 0,5 e uma percentagem de $40 \%$ de $\mathrm{C}$ na matéria seca vegetal, estimou-se uma adição de $\mathrm{C}$ ao solo $15 \%$ maior no $\mathrm{PD}\left(2,3 \mathrm{Mg} \mathrm{ha}^{-1} \mathrm{ano}^{-}\right.$ $\left.{ }^{1}\right)$ do que no PC $\left(2,0 \mathrm{Mg} \mathrm{ha}^{-1} \mathrm{ano}^{-1}\right)$. A maior adição anual de $\mathrm{C}$ via resíduos vegetais, associado à manutenção dos resíduos vegetais na superfície do solo, o que diminui a taxa de decomposição microbiana, resultou num acúmulo diferencial de $3,2 \mathrm{Mg} \mathrm{ha}^{-1} \mathrm{de}$ COT e $1,2 \mathrm{Mg} \mathrm{ha}^{-1} \mathrm{de} \mathrm{COP}$ na camada de $0-20 \mathrm{~cm}$ do solo em PD, em comparação ao solo em PC (Tabela 1). Em comparação aos valores de 0,3 a $0,7 \mathrm{Mg} \mathrm{ha}^{-1}$ ano $^{-1}$ determinados em Argissolos do RS (BAYER et al., 2000; AMADO et al., 2001), a taxa de incremento do COT $\left(0,15 \mathrm{Mg} \mathrm{ha}^{-1}\right.$ ano $\left.^{-1}\right)$ neste solo sob PD pode ser considerada baixa, o que provavelmente esteja relacionado à sua textura argilosa e mineralogia predominantemente gibsítica. Estes fatores determinam uma alta proteção física da MO no solo, mesmo sob preparo convencional.

O COP representou de 24 a $31 \%$ do COT (Tabela 1). Esses valores podem ser considerados baixos em comparação a valores em torno de $50 \%$ verificados em solos de regiões frias e ou semiáridas (CAMBARDELLA \& ELLIOT, 1992; CHAN, 1997), e refletem as condições de temperatura e umidade altamente favoráveis à atividade microbiana nas condições de clima subtropical úmido do presente estudo. Por outro lado, o aumento relativo nos estoques de COP foi maior do que o de COT, demonstrando que a fração lábil da $\mathrm{MO}$ foi mais sensível às mudanças do manejo do que a MO total. As maiores alterações nos estoques de $\mathrm{C}$ ocorreram na camada superficial do solo $(0-5 \mathrm{~cm})$, onde o COP apresentou-se 58\% superior no PD em relação ao PC, enquanto o COT, apenas $32 \%$. Por outro lado, a incorporação dos resíduos vegetais no PC resultou em maiores estoques de COT e COP na camada mais profunda (10-20cm), em comparação ao solo em PD.

$\mathrm{O}$ diâmetro médio geométrico (DMG) dos agregados variou de 1,6 a $3,7 \mathrm{~mm}$ no solo em $\mathrm{PD}$, e de 1,6 a 2,3mm no solo em PC (Tabela 1 ). O efeito do PD na estabilidade de agregados foi mais pronunciado na camada superficial $(0-5 \mathrm{~cm})$ do solo, e altamente relacionado aos teores de COT e COP do solo [ DMG $(\mathrm{mm})=-4,79+0,19 \operatorname{COT}\left(\mathrm{g} \mathrm{dm}^{-3}\right), \mathrm{r}^{2}=0,97, \mathrm{P}<0,01$; $\operatorname{DMG}(\mathrm{mm})=-1,07+0,35 \mathrm{COP}\left(\mathrm{g} \mathrm{dm}^{-3}\right), \mathrm{r}^{2}=0,77, \mathrm{P}<0,02$ ]. Esses resultados concordam com CARPENEDO \& MIELNICZUK (1990) que encontraram efeito semelhante em Latossolos do RS. Por sua vez, CHAN (1997) verificou efeito diferenciado de frações orgânicas na estabilidade de agregados de Vertissolos. No presente estudo verificou-se que, para cada aumento de uma unidade no COP, ocorreu um aumento de 0,35 unidades no DMG dos agregados, enquanto a mesma variação no COT resultou numa variação positiva de apenas 0,19 unidades no DMG. A maior labilidade do COP como fonte de $\mathrm{C}$ e energia aos microrganismos possivelmente refletiu-se de forma mais expressiva na estabilidade, principalmente, de macroagregados. Por sua vez, o COT engloba uma expressiva fração de matéria orgânica humificada, a qual atua basicamente na estabilidade de microagregados.

O não revolvimento do solo e a adição dos resíduos das culturas na superfície do solo em PD desencadeia inúmeros processos físicos, químicos e biológicos fortemente interrelacionados, geralmente sinérgicos entre si, como é o caso dos processos que levam ao aumento da estabilidade dos agregados e dos estoques de matéria orgânica no solo. O maior carbono orgânico determina maior estabilidade de agregados, e esta, por sua vez, maior proteção física da matéria orgânica. Estabelece-se, assim, uma relação causa-efeito entre agregação e a matéria orgânica (SIX et al., 1999), na qual o aumento da estabilidade de agregados do 
Tabela 1 - Conteúdo de carbono orgânico total (COT) e particulado (COP) de um Latossolo Bruno cultivado, durante 21 anos, nos sistemas de preparo convencional (PC) e plantio direto (PD).

\begin{tabular}{|c|c|c|c|c|c|c|c|c|c|c|}
\hline \multirow[t]{2}{*}{ Sistema } & \multicolumn{2}{|c|}{ COT } & \multicolumn{2}{|c|}{ COP } & \multicolumn{2}{|c|}{$\Delta \mathrm{COT}$} & \multicolumn{2}{|c|}{$\Delta \mathrm{COP}$} & \multirow{2}{*}{$\frac{(\mathrm{COP} / \mathrm{COT}) \times 100}{\%}$} & \multirow{2}{*}{$\begin{array}{c}\mathrm{DMG} \\
\mathrm{mm}\end{array}$} \\
\hline & $\mathrm{g} \mathrm{dm}^{-3}$ & $\mathrm{Mg} \mathrm{ha}^{-1}$ & $\mathrm{~g} \mathrm{dm}^{-3}$ & $\mathrm{Mg} \mathrm{ha}^{-1}$ & $\mathrm{Mg} \mathrm{ha}^{-1}$ & $\%$ & $\mathrm{Mg} \mathrm{ha}^{-1}$ & $\%$ & & \\
\hline \multicolumn{11}{|l|}{$0-5 \mathrm{~cm}$} \\
\hline $\mathrm{PC}$ & $33,4 \mathrm{~b}$ & 16,7 & $8,60 \mathrm{~b}$ & 4,3 & & & & & 26 & $1,6 \mathrm{~b}$ \\
\hline PD & $44,0 \mathrm{a}$ & 22,0 & $13,6 \mathrm{a}$ & 6,8 & 5,3 & 32 & 2,5 & 58 & 31 & $3,7 \mathrm{a}$ \\
\hline \multicolumn{11}{|l|}{$5-10 \mathrm{~cm}$} \\
\hline $\mathrm{PC}$ & $34,6 \mathrm{a}$ & 17,3 & $8,6 \mathrm{a}$ & 4,3 & & & & & 25 & $1,9 \mathrm{~b}$ \\
\hline PD & $37,4 \mathrm{a}$ & 18,7 & $8,8 \mathrm{a}$ & 4,4 & 1,4 & 8 & 0,1 & 2 & 24 & $2,8 \mathrm{a}$ \\
\hline \multicolumn{11}{|c|}{$10-20 \mathrm{~cm}$} \\
\hline $\mathrm{PC}$ & $36,2 \mathrm{a}$ & 36,2 & $9,6 \mathrm{a}$ & 9,6 & & & & & 27 & $2,3 \mathrm{a}$ \\
\hline PD & $32,7 \mathrm{a}$ & 32,7 & $8,2 \mathrm{a}$ & 8,2 & $-3,5$ & -10 & $-1,4$ & -15 & 25 & $1,6 \mathrm{~b}$ \\
\hline
\end{tabular}

$\Delta \mathrm{COT}\left(\mathrm{Mg} \mathrm{ha}^{-1}\right)=\mathrm{PD}-\mathrm{PC} ; \Delta \mathrm{COT}(\%)=[(\mathrm{PD}-\mathrm{PC}) / \mathrm{PC}] \times 100$.

Médias não seguidas de letras iguais na coluna diferem entre si pelo teste de Tukey em nível de 5\% de probabilidade de erro.

solo é, ao mesmo tempo, uma causa e uma consequiência dos maiores estoques de matéria orgânica do solo. Por sua vez, a quebra dos agregados de solo no PC expõe a matéria orgânica à atividade microbiana, reduzindo o conteúdo de carbono orgânico do solo.

A relação entre a matéria orgânica e o processo de agregação das partículas de solo, ou vice-versa, é complexa. Contudo através do uso de técnicas de fracionamento físico tem sido possível determinar o comportamento de compartimentos dessa complexidade, o que tem contribuído no seu melhor entendimento. Adicionalmente, pressupõe-se que, associada ao fracionamento físico da matéria orgânica, a aplicação de técnicas espectroscópicas para o estudo qualitativo das frações orgânicas pode contribuir para a melhor compreensão da dinâmica da matéria orgânica, principalmente no que se refere à sua proteção física em solos tropicais e subtropicais sob diferentes sistemas de manejo do solo.

\section{REFERÊNCIAS BIBLIOGRÁFICAS}

AMADO, T.J.C. et al. Potencial de culturas de cobertura em acumular carbono e nitrogênio no solo no plantio direto e a melhoria da qualidade ambiental. R Bras Ci Solo, v.25, p.189-197, 2001.

BALESDENT, J.; CHENU, C.; BALABANE, M. Relationship of soil organic matter dynamics to physical protection and tillage. Soil Till Res, v.53, p. $215-230,2000$

BAYER, C. et al. Organic matter storage in a sandy clay loam Acrisol affected by tillage and cropping systems in southern Brazil. Soil Till Res, v.54, p.101-109, 2000.

CAMBARDELlA, C.A.; ELliOT, E.T. Particulate soil organic-matter changes across a grassland cultivation sequence. Soil Sci Soc Am J , v.56, p.777-783, 1992.

CARPENEDO, V.; MIELNICZUK, J. Estado de agregação e qualidade de agregados de Latossolos Roxos, submetidos a diferentes sistemas de manejo. R Bras Ci Solo, v.14, p.99$105,1990$.

CHAN, K.Y. Consequences of changes in particulate organic carbon in vertisols under pasture and cropping. Soil Sci Soc Am J, v.61, p.1376-1382, 1997.

EMBRAPA-EMPRESA BRASILEIRA DE PESQUISA AGROPECUÁRIA. Centro Nacional de Pesquisa de Solos. Manual de métodos de análise de solo. 2.ed. rev. atual. Rio de Janeiro, 1997. 212p.

KEMPER, W.D.; CHEPIL, W.S. Size distribution of aggregates. In: BLACK, C.A.; EVANS, D.D.; WHITE, J.L. (Eds). Methods of soil analysis. Madison: American Society of Agronomy, 1965. p.499-510. (Agronomy, Monogr., 9).

SIX, J.; ELliOT, E.T.; PAUSTIAN, K. Aggregate and organic matter dynamics under conventional and notillage systems. Soil Sci Soc Am J, v.63, p.1350-1358, 1999. 